\title{
TNAURice: Database on rice varieties released from Tamil Nadu Agricultural University
}

\author{
Jegadeesan Ramalingam ${ }^{1}{ }^{*}$, Loganathan Arul ${ }^{1}$, Natarajan Sathishkumar ${ }^{1}$, \\ Dhandapani Vignesh $^{1}$, Katiannan Thiyagarajan ${ }^{2}$, Ramasamy Samiyappan ${ }^{1}$
}

${ }^{1}$ Centre for Plant Molecular Biology, Tamil Nadu Agricultural University, Coimbatore - 641 003, India; ${ }^{2}$ Centre for Plant Breeding and Genetics, Tamil Nadu Agricultural University, Coimbatore - 641 003, India; Jegadeesan Ramalingam - Email: jrjagadish@tnau.ac.in; Phone: 914226611353 ; *Corresponding author

Received July 26, 2010; Accepted August 26, 2010; November 27, 2010

Abstract:

We developed, TNAURice: a database comprising of the rice varieties released from a public institution, Tamil Nadu Agricultural University (TNAU), Coimbatore, India. Backed by MS-SQL, and ASP-Net at the front end, this database provide information on both quantitative and qualitative descriptors of the rice varities inclusive of their parental details. Enabled by an user friendly search utility, the database can be effectively searched by the varietal descriptors, and the entire contents are navigable as well. The database comes handy to the plant breeders involved in the varietal improvement programs to decide on the choice of parental lines. TNAURice is available for public access at http://www.btistnau.org/germdefault.aspx.

\section{Background:}

Rice (Oryza sativa L.) is the staple food for more than three billion people, over half the world's population. It provides $27 \%$ of dietary energy and $20 \%$ of dietary protein in the developing world. About $90 \%$ of the world's rice is grown and consumed in Asia [1]. Research into rice is crucial for the development of technologies that will increase the productivity for farmers who rely on rice for their livelihood. Research in rice is aimed at increasing the yield, yield stability and improving resistance against abiotic and biotic stresses. There exist a huge collection of rice germplasm carrying useful genes for the above traits and have been continuously exploited by the plant breeders towards developing improved rice varieties. Detailed documentation of the germplasm/varieties facilitated by an easy and efficient search and retrieval system is an absolute necessity considering the wide range of breeding activities taken up globally in rice, and other crops. There are a select public/private databases carrying molecular and genetic data of specific model species such as maize [2] Medicago [3], rice [4] and Arabidopsis [5] which caters to more of an investigative nature of research. However, when it comes to applied varietal developmental programs, familiarity of the plant breeders on aspects such as, pedigree, yield, resistance levels to biotic and abiotic stresses etc., is important for evolving a successful hybridization program. TNAURice is an initititve in this direction to serve as ready reckoner to the rice breeders, it is a compilation of the rice varieties developed from Tamil Nadu Agricultural University, Coimbatore, one of the premier institutes engaged in rice research for the past 100 years in India. About two hundred and fifty rice varieties developed using different breeding approaches from various research stations of TNAU are included in this database. Here, we describe the construction and utility of TNAURice, this database will be of use to the plant researchers in general and rice breeders in particular towards successful planning of their breeding objectives.
Methodology of development:

The data pertaining to the rice cultivars had been collected from records maintained at the Paddy Breeding Station (PBS), Coimbatore and from the constituent research stations of TNAU involved in rice breeding program. The varieties included in the database are from the following research stations located at different places in the state of Tamil Nadu: Coimbatore, Aduthurai, Ambasamudram, Tirurkuppam, Talainayar, Palur, Paramakudi, Thirupathisaram, Madurai, Pondicherry, Paiyur and Trichy. The rice varieties are identified by the following 29 descriptors: Name, Parentage, Duration, Season, Area of adoption, Yield(tha), Habit, Leaf sheath, Axil, Junction, Auricle, ligule, Septum, Leaf blade, Flag leaf, Fertile glumes, Apiculus, Awns, Panicle, Exsertion, Grain: $\mathrm{L}^{*} \mathrm{~B} * \mathrm{~T}(\mathrm{~mm}), 1000$ grain weight(g), Brown rice: $\mathrm{L}^{*} \mathrm{~B} * \mathrm{~T}(\mathrm{~mm}), \mathrm{L} / \mathrm{B}$ ratio, Rice Grade, Rice colour, Abdominal white, Transluency, Hulling(\%), Milling(\%) and Protein content.

The TNAU rice database was structured using Microsoft Structured Query Language (MS-SQL). The user interface of the database was enabled with Microsoft ASP.NET, a standard generalized and most advanced web development application. The home page contains the textual information organized under "Home, About database, Link outs and Help desk"

(Figure 1). The information available under the above mentioned subcomponents was compiled separately in an abridged form. A navigable link to the data is enabled under "Research stations", the varieties developed in each of the research stations were listed, accordingly. Keyword based searching of the data is enabled for the following eleven varietal descriptors such as "Varieties, Popular name, Parentage, Duration, Season, Area of adoption, Habit, Awns, Panicle, Rice grade and Rice colour" as listed in the search window. 


\section{Bioinformation}

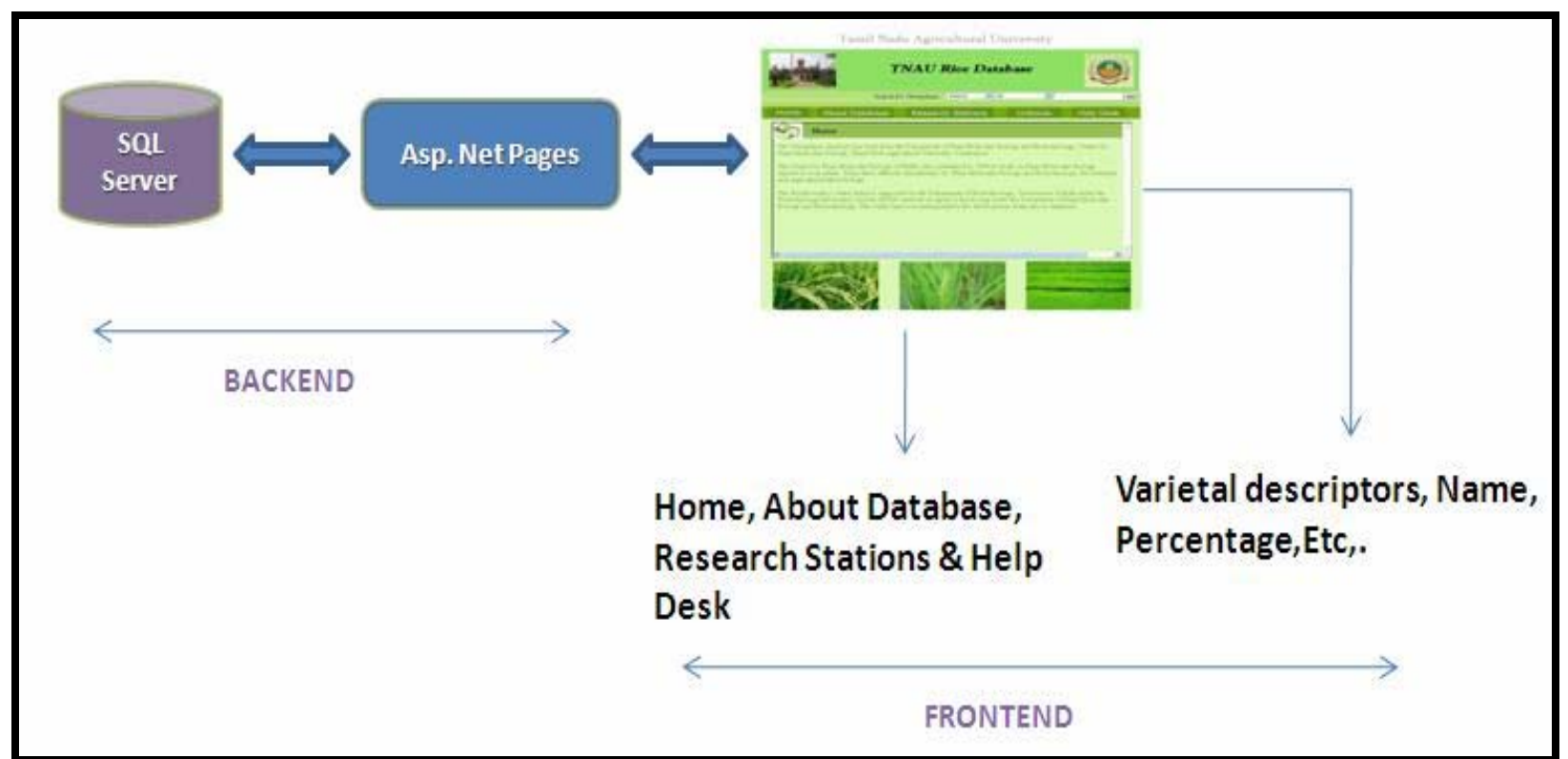

Figure 1: TNAURice architecture showing the network of front and back end of the database

\section{Utility:}

The use of database technologies has drawn the attention of a subset of the biological community, as of now it is limited to a small sector of the scientific community. However, more and more individuals/institutions are generating experimental data on a large scale and are in need of developing and managing databases of their own. Effective use of information may strongly promote biological studies, and may lead to many important findings [6]. Plant germplasm resources play an important role in crop improvement programs [7]. The use and benefits derived from conserved germplasm is the sole criterion for assessing the genetic conservation program of a crop. The use of rice germplasm includes both direct use in rice production and indirect use in rice breeding programs as parents. Presently, information regarding the available and evaluated germplasm is rarely documented in the form of a well structured database. It is therefore, time-consuming and laborious for individual researchers to extract and organize varietal information from sources such as, notebooks/records or from typed electronic pages. Thus the primitive nature of documentation of the varietal information is a major limitation and restricts its utility by time and space. This draws an urgent need to consolidate rice germplasm through a database system [8]. The present database is well structured, comprehensive and complete with respect to the details of rice varieties that have been released from TNAU till date. It is aimed at the Indian and International rice researchers and the progressive farming community. In future, we plan to include rice varieties and hybrids released from other Indian agricultural institutions as well.

\section{Acknowledgement:}

This work is supported by the Department of Biotechnology, Government of India under Biotechnology Information System (BTIS) scheme.

\section{References:}

[1] RS Zeigler \& A Barclay. Rice 1:3 (2008)

[2] CJ Lawrence et al. Plant Physiol 138: 55 (2005) [PMID :15888678]

[3] SB Cannon et al. Plant Physiol 138: 38 (2005) [PMID : 15888676]

[4] K Sakata et al. Nucleic Acids Res 30: 98 (2002)

[5] RL Poole. Methods Mol Biol 406:179 (2007) [PMID :18287693]

[6] Y Yamazaki \& P Jaiswal. Plant Cell Physiol 46: 63 (2005) [PMID:15659431]

[7] K Okuno. Proceedings of the International symposium on rice germplasm evaluation and enhancement. 195: 101 (1998)

[8] M Rai. Proceedings of the International Symposium on Rice Germplasm Evaluation and Enhancement 195: 83 (1998)

Edited by $P$ Kangueane

Citation: Ramalingam et al. Bioinformation 5(6): 264- 265 (2010)

License statement: This is an open-access article, which permits unrestricted use, distribution, and reproduction in any medium, for non-commercial purposes, provided the original author and source are credited. 